\title{
Acute Generalized Exanthematous Pustulosis in Close Temporal Association with mRNA-1273 Vaccine
}

Fouad MITRI, Ferdinand TOBERER, Alexander H. ENK and Martin HARTMANN

Department of Dermatology, University Hospital Heidelberg, DE-69120 Heidelberg, Germany. E-mail: fouad.Mitri@med.uni-heidelberg.de Accepted Oct 28, 2021; Epub ahead of print Oct 28, 2021

We report here a case of a patient who developed pustules and skin changes all over his body 8 days after receiving the Moderna COVID-19 vaccine. The patient was otherwise healthy and was not on any medication prior to vaccination. It is notable that he also had a pre-existing disease that causes jaundice, which makes the skin yellow. The rash he developed is usually a serious adverse event against medications, but it can also occur after infections. The patient was treated with cortisone and the rash started to improve. It is important to note that this type of rash has rarely been reported after COVID vaccines.

\section{CASE REPORT}

A 27-year-old man presented to our outpatient clinic with an erythematous, pustulo-papular rash that had developed in the past 4 days. The lesions appeared rapidly, initially involving the thorax than the extremities, and, finally, the neck, ears, and paranasal area. Eight days earlier, the patient had received the first dose of the Moderna mRNA-1273 coronavirus disease 2019 (COVID-19) vaccine. He reported having no prior medications, allergies, fever, or recent travel. The patient had not undergone recent dental procedures or taken natural remedies. His past medical history includes Gilbert's syndrome. Physical examination revealed a widespread erythematous rash with multiple, mostly non-follicular, pustules. Furthermore, jaundice was noted on the back and upper chest (Fig. 1). Laboratory results revealed neutrophilia $\left(10.11 \times 10^{9} / 1\right)$ as well as hyperbilirubinaemia $(6.6$ $\mathrm{mg} / \mathrm{dl}$ ) without further pathologies. Immunoglobulin Ms (IgMs) for Epstein-Barr virus and cytomegalovirus were negative. An abdominal ultrasound did not reveal any significant findings. Histopathology revealed superficial epidermal pustulation, neutrophilic

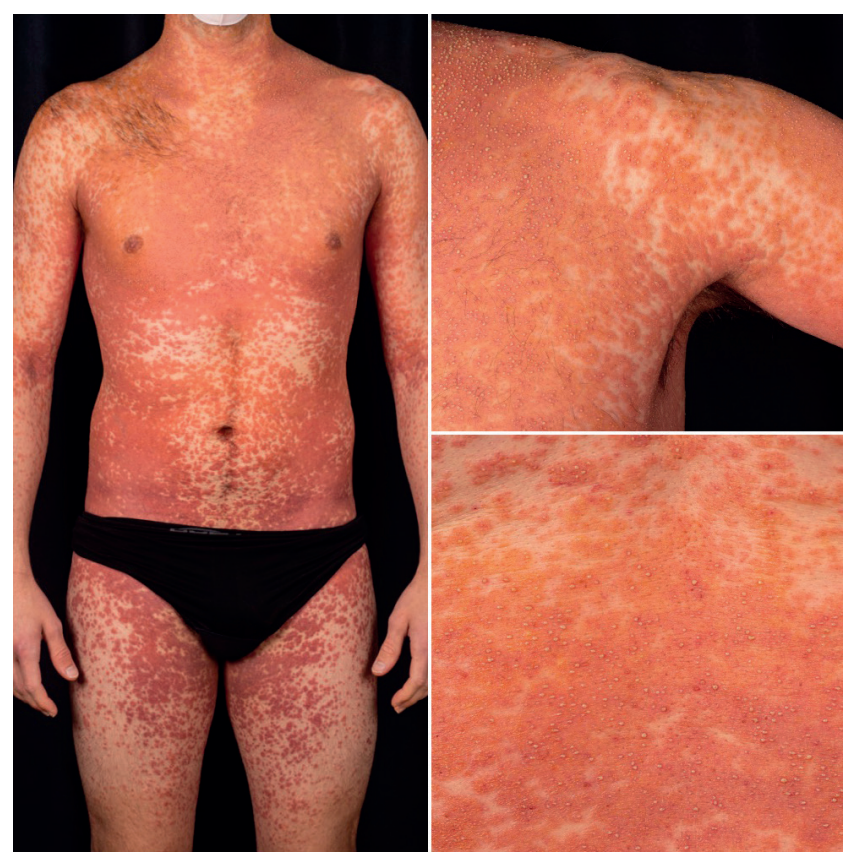

Fig. 1. Clinical findings. Panoramic and close-up views showing a widespread erythematous rash with multiple pustules. Note the icteric component on the upper breast.

spongiosis, and a mixed inflammatory cell infiltrate consisting of neutrophils, eosinophils and lymphocytes in the upper dermis (Fig. 2). Based on the clinical and histopathological findings, acute generalized exanthematous pustulosis (AGEP) was diagnosed. The patient was initially treated with topical glucocorticoids, followed by $80 \mathrm{mg}$ prednisolone orally for 3 days. After a few days, his skin improved significantly.

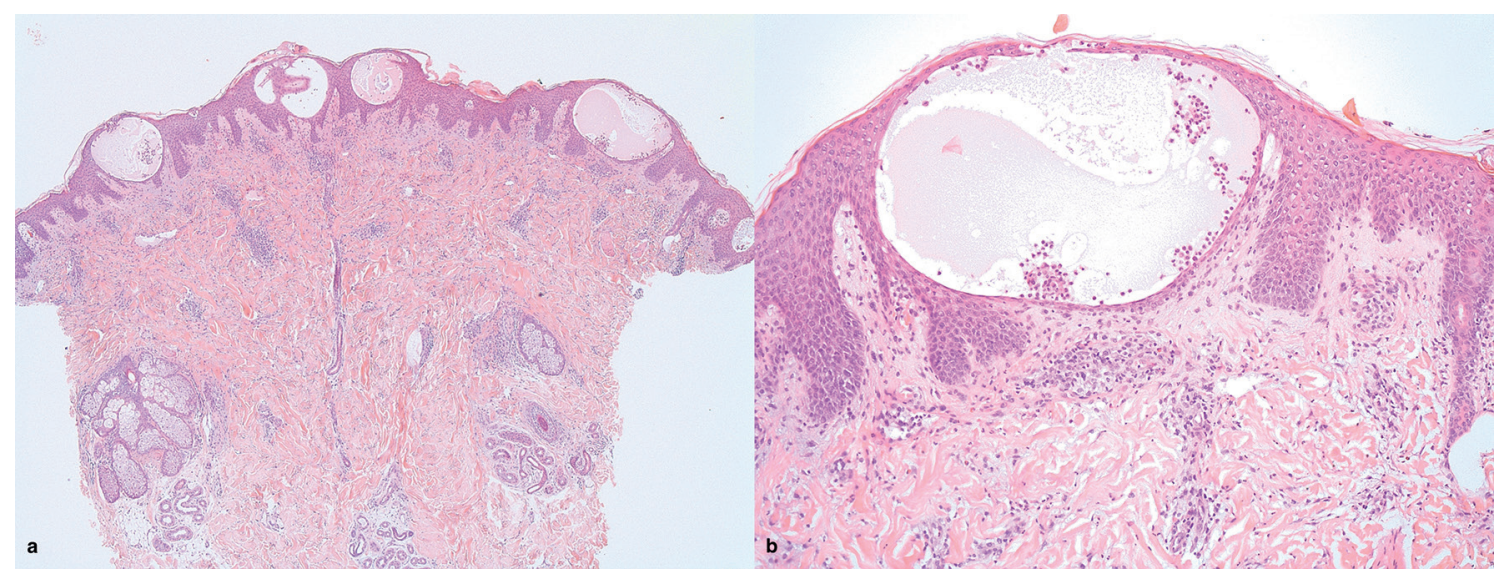

Fig. 2. Histopathology. (a) Section showing superficial epidermal pustulation (haematoxylin and eosin (H\&E)-stain, original magnification $\times 25$ ). (b) Close-up view revealing neutrophilic spongiosis and a mixed cell dermal infiltrate (H\&E stain, original magnification $\times 100$ ). 


\section{DISCUSSION}

AGEP is a severe cutaneous adverse reaction with rapid onset (1). It is usually drug-induced and is rarely of infectious aetiology. It is characterized by an acute rash with pustules on an erythematous/oedematous base. Fever, lymphocytosis, neutrophilia, and eosinophilia are also common manifestations (2). Systemic involvement, including hepatic, renal, or pulmonary insufficiency, occurs in approximately $20 \%$ of cases (1). Histology typically shows spongiform subcorneal pustules, an oedematous dermis, and perivascular infiltrates with neutrophils and eosinophils (3).

AGEP is often a self-limiting condition. Therapy usually involves removing the triggering drug, in some cases, topical steroids and disinfectants are applied and in extensive disease, systemic steroids can be given for a short time (4).

Interestingly, AGEP has been described recently in association with severe acute respiratory syndrome coronavirus-2 (SARS-CoV-2) infections (5), mainly through the use of COVID-19 drugs (such as hydroxychloroquine and lopinavir/ritonavir) (6) or antibiotics. Moreover, AGEP has been described after SARS-CoV2 vaccines, including Johnson and Johnson's Janssen Ad26.COV2.S (7), ChAdOx1 nCoV-19 vaccine, AstraZeneca ${ }^{\circledR}$ (8) and, recently, mRNA 1273 vaccine, Moderna (9). To our knowledge, this is the second case of AGEP in close temporal proximity to an mRNA-1273 vaccine.

Studies are needed to generate solid guidelines regarding further vaccinations. Our recommendation to the patient was to take the second dose using another mRNA vaccine, preferably the Pfizer-BioNTec ${ }^{\circledR}$.

\section{REFERENCES}

1. Szatkowski J, Schwartz RA. Acute generalized exanthematous pustulosis (AGEP): a review and update. J Am Acad Dermatol 2015; 73: 843-848.

2. Hadavand MA, Kaffenberger B, Trinidad JC. Clinical presentation and management of atypical and recalcitrant acute generalized exanthematous pustulosis (AGEP). J Am Acad Dermatol 2020 Sep 11. [Epub ahead of print].

3. Kardaun SH, Kuiper H, Fidler V, Jonkman MF. The histopathological spectrum of acute generalized exanthematous pustulosis (AGEP) and its differentiation from generalized pustular psoriasis. J Cutan Pathol 2010; 37: 1220-1229.

4. Beltraminelli HS, Lerch M, Arnold A, Bircher AJ, Haeusermann P. Acute generalized exanthematous pustulosis induced by the antifungal terbinafine: case report and review of the literature. Br J Dermatol 2005; 152: 780-783.

5. Pezzarossa E, Ungari M, Caresana G, Sagradi F, Cimardi L, Pan $A$, et al. Acute generalized exanthematous pustulosis (AGEP) in 12 patients treated for SARS-CoV-2 positive pneumonia. Am J Dermatopathol 2021; 43: 342-348.

6. Nobari NN, Seirafianpour F, Mashayekhi F, Goodarzi A. A systematic review on treatment-related mucocutaneous reactions in COVID-19 patients. Dermato Ther 2021; 34 : e14662.

7. Lospinoso K, Nichols CS, Malachowski SJ, Mochel MC, Nutan F. A case of severe cutaneous adverse reaction following administration of the Janssen Ad26.COV2.S COVID-19 vaccine. JAAD Case Rep 2021; 13: 134-137.

8. Kang SY, Park SY, Kim JH, Lee SM, Lee SP. COVID-19 vaccineinduced acute generalized exanthematous pustulosis. Korean J Intern Med 2021; 36: 1537-1538.

9. Agaronov A, Makdesi C, Hall CS. Acute generalized exanthematous pustulosis induced by Moderna COVID-19 messenger RNA vaccine. JAAD Case Rep 2021; 16: 96-97. 